\title{
Clustering of matter in waves and currents
}

\author{
Marija Vucelja ${ }^{1}$, Gregory Falkovich ${ }^{1}$, and Itzhak Fouxon ${ }^{1,2}$ \\ 1 Physics of Complex Systems, Weizmann Institute of Science, Rehovot 76100, Israel. \\ ${ }^{2}$ Racah Institute of Physics, Hebrew University of Jerusalem, Jerusalem 91904, Israel.
}

(Dated: April 9, 2018)

\begin{abstract}
The growth rate of small-scale density inhomogeneities (the entropy production rate) is given by the sum of the Lyapunov exponents in a random flow. We derive an analytic formula for the rate in a flow of weakly interacting waves and show that in most cases it is zero up to the fourth order in the wave amplitude. We then derive an analytic formula for the rate in a flow of potential waves and solenoidal currents. Estimates of the rate and the fractal dimension of the density distribution show that the interplay between waves and currents is a realistic mechanism for providing patchiness of pollutant distribution on the ocean surface.
\end{abstract}

PACS numbers: 47.27.Qb, 05.40.-a

Random compressible flows produce very inhomogeneous distribution of density, see [1, 2, 3, 4, 4, 5, 6, 7, 8, 9] for theory and 10, 11, 12, 13, 14, 15, 16] for experiments. Here we study the growth of density inhomogeneities at small scales, where the flow can be considered spatially smooth. It can then be characterized by the Lyapunov exponents whose sum is the logarithmic rate of change of an infinitesimal volume element, that is minus the density rate of change $\lambda$. It is called also the entropy production rate or the clustering rate. Since contracting regions contain more statistical weight than expanding ones, $\lambda$ is generally positive in a random compressible flow [2, 3, 4, 5] (an analog of the second law of thermodynamics). As a result, density grows on most trajectories. In the limit of infinite time, density concentrates on a constantly evolving fractal set characterized by a singular (Sinai-Ruelle-Bowen) measure [4, 17, 18, 19]. Our goal here is to establish what determines the rate $\lambda$ in fluid flows with waves, particularly, on liquid surfaces. Patchiness in the distribution of litter on the surface of lakes and pools and of oil slicks and seaweeds on the sea surface is well-known empirically while there is no theory that describes it. We think that in many situations patchiness is a signature of a fractal measure forming on the surface. Our purpose here is to estimate how fast this fractal set is formed and what is its fractal dimension.

Any velocity field can be separated into solenoidal (incompressible) and potential components. Surface flows can be compressible, even for incompressible fluids. For example, underwater turbulence produces compressible surface currents that lead to fractal distributions of surface density [11, 12, 13, 15, 16, 20]. However, underwater turbulence is relatively rare in natural environment (because of stable stratification) and large-scale currents are usually incompressible. Compressible component of the surface flows is then provided by potential waves. Linear waves just oscillate, net effects are produced by nonlinearity. Every running plane wave provides for a (Stokes) drift proportional to the square of the wave amplitude. A set of random waves provides for the Lyapunov exponents proportional to the fourth power of the wave amplitudes yet the sum of the exponents $-\lambda$ is found to be zero for purely potential waves with Gaussian statistics (nonzero rate appears only in the sixth order in wave amplitudes, i.e. it is so small as to be practically unobservable in most cases) [8]. Here we use the recently derived general formula for the entropy production rate [21] and show that the account of wave interaction (which makes the statistics weakly non-Gaussian) does not bring non-zero $\lambda$ in the fourth order in wave amplitudes. We then suggest that in many situations (particularly on liquid surfaces) the growth of density inhomogeneities is due to an interplay between potential waves and solenoidal currents. For such flows, we calculate $\lambda$ and the fractal dimension of the resulting measure and consider different limits.

In the velocity field $\boldsymbol{v}(t, \boldsymbol{x})$, the trajectory $\boldsymbol{X}(t, \boldsymbol{x})$ satisfies the equation $\partial_{t} \boldsymbol{X}=\boldsymbol{v}(t, \boldsymbol{X})$ with the initial condition $\boldsymbol{X}(0, \boldsymbol{x})=\boldsymbol{x}$. The rate of density change along the trajectory averaged over $\boldsymbol{x}$ is given by [21]:

$$
\lambda=-\lim _{t \rightarrow \infty}\langle w(t, \boldsymbol{X})\rangle=\int_{0}^{\infty} d t\langle w(0, \boldsymbol{x}) w(t, \boldsymbol{X})\rangle,
$$

with $w \equiv \boldsymbol{\nabla} \cdot \boldsymbol{v}$. This is a generalization of the Kawasaki formula 22] (obtained in the context of statistical physics) to time-dependent flows with a steady statistics.

For a general flow, it is impossible to relate the Lagrangian integral (10) to the velocity spectra or correlation functions given usually in the Eulerian frame. However, for low-amplitude waves, fluid particles shift little during a period, which allows for an analytical treatment. Considering waves with the dispersion relation $\Omega_{\boldsymbol{k}}$ and packets with both the wavenumber and the width of order $k$, we estimate the correlation time of $w$ as $\Omega_{\boldsymbol{k}}^{-1}$ and the correlation length as $k^{-1}$. The deviation $\boldsymbol{X}(t, \boldsymbol{x})-\boldsymbol{x}$ during $t \simeq \Omega_{\boldsymbol{k}}^{-1}$ is $\epsilon=k v / \Omega_{\boldsymbol{k}} \ll 1$ times smaller than $k^{-1}$. We now expand (11) near $\boldsymbol{x}$ up to $\epsilon^{4}$ :

$$
\begin{aligned}
\lambda & \approx \int_{0}^{\infty} d t\left[\langle w(0) w(t)\rangle+\left\langle w(0) \frac{\partial w(t)}{\partial x^{\alpha}} \int_{0}^{t} d t_{1} v^{\alpha}\left(t_{1}\right)\right\rangle\right. \\
& +\left\langle w(0) \frac{\partial w(t)}{\partial x^{\alpha}} \int_{0}^{t} d t_{1} \frac{\partial v^{\alpha}\left(t_{1}\right)}{\partial x^{\beta}} \int_{0}^{t_{1}} d t_{2} v^{\beta}\left(t_{2}\right)\right\rangle \\
& \left.+\frac{1}{2}\left\langle w(0) \frac{\partial w(t)}{\partial x^{\beta} \partial x^{\alpha}} \int_{0}^{t} d t_{1} v^{\alpha}\left(t_{1}\right) \int_{0}^{t} d t_{2} v^{\beta}\left(t_{2}\right)\right\rangle\right] .
\end{aligned}
$$


All quantities here are taken at the same point in space.

We start the consideration of (2) from the simplest case when the flow is solely due to weakly nonlinear waves. The normal coordinates of such waves satisfy the equation $\partial_{t} a_{\boldsymbol{k}}=-i \delta \mathcal{H} / \delta a_{k}^{*}$ while the velocity Fourier component is assumed to be given by $\boldsymbol{v}_{\boldsymbol{k}}=\boldsymbol{A}_{\boldsymbol{k}}\left(a_{\boldsymbol{k}}-a_{-\boldsymbol{k}}^{*}\right)$. Here $\mathcal{H}$ is the wave Hamiltonian, which can be expanded in wave amplitudes as follows [23]: $\mathcal{H}=\int d \boldsymbol{k} \Omega_{\boldsymbol{k}}\left|a_{\boldsymbol{k}}\right|^{2}+$ $\frac{1}{2} \int d \boldsymbol{k}_{123}\left(\boldsymbol{V}_{123} a_{1} a_{2}^{*} a_{3}^{*}+\right.$ c.c. $)+\cdots$. We do not write explicitly here other (third and fourth-order) terms since they will not contribute $\lambda$ up to $\sim \epsilon^{4}$. We use throughout the shorthand notations $\boldsymbol{V}_{123}=V_{123} \delta\left(\boldsymbol{k}_{1}-\boldsymbol{k}_{2}-\boldsymbol{k}_{3}\right)$ and $V_{123}=V\left(\boldsymbol{k}_{1}, \boldsymbol{k}_{2}, \boldsymbol{k}_{3}\right), \Omega\left( \pm \boldsymbol{k}_{i}\right)=\Omega_{ \pm i}$ and $\boldsymbol{A}\left(\boldsymbol{k}_{i}\right)=\boldsymbol{A}_{i}$.

One derives the clustering rate up to $\epsilon^{4}$ using a standard perturbation theory for weakly interacting waves [23]. The first term in (2) is the time integral (the zerofrequency value) of the second moment. At the order $\epsilon^{2}$, the second moment in the frequency representation is proportional to the delta function: $\left\langle a^{*}(\boldsymbol{k}, \omega) a\left(\boldsymbol{k}^{\prime}, \omega^{\prime}\right)\right\rangle=$ $(2 \pi)^{d+2} n(\boldsymbol{k}) \delta\left(\omega-\Omega_{\boldsymbol{k}}\right) \delta\left(\boldsymbol{k}-\boldsymbol{k}^{\prime}\right) \delta\left(\omega-\omega^{\prime}\right)$. A finite width over $\omega$ and a finite value at $\omega=0$ appear either due to finite linear attenuation (the case considered in [24]) or due to nonlinearity in the second order of perturbation theory (which gives $\epsilon^{4}$ and is considered here). The second term in (2) is the triple moment which appears in the first order of the perturbation theory and the last two terms contain the fourth moment which is to be taken at the zeroth order (i.e. as a product of two second moments). Straightforward calculations then give for weakly nonlinear waves the $\epsilon^{4}$ contribution:

$$
\begin{aligned}
& \lambda=\operatorname{Re} \int \frac{d \boldsymbol{k}_{2} d \boldsymbol{k}_{3}}{(2 \pi)^{2 d}} \delta\left(\Omega_{2}-\Omega_{3}\right) n\left(\boldsymbol{k}_{2}\right) n\left(\boldsymbol{k}_{3}\right)\left\{\int \frac{d \boldsymbol{k}_{1}}{(2 \pi)^{d}}\right. \\
& \times\left[( \frac { \boldsymbol { V } _ { 2 1 3 } ^ { * } } { \Omega _ { 1 } } - \frac { \boldsymbol { V } _ { 3 - 1 2 } } { \Omega _ { - 1 } } ) \left((2 \pi)^{3 d+1}\left|\boldsymbol{A}_{1} \cdot \boldsymbol{k}_{1}\right|^{2} \frac{V_{213}}{\Omega_{1}}\right.\right. \\
& \left.\left.-\frac{(2 \pi)^{2 d}}{\Omega_{2}}\left(\boldsymbol{A}_{1}^{*} \cdot \boldsymbol{k}_{1}\right)\left(\boldsymbol{A}_{2} \cdot \boldsymbol{k}_{2}\right)\left(\boldsymbol{A}_{3}^{*} \cdot\left(\boldsymbol{k}_{2}+\boldsymbol{k}_{1}\right)\right)\right)\right]+ \\
& \left.+\frac{\pi}{\Omega_{2}^{2}}\left|\left(\boldsymbol{A}_{3} \cdot \boldsymbol{k}_{3}\right)\left(\boldsymbol{A}_{2}^{*} \cdot \boldsymbol{k}_{3}\right)-\left(\boldsymbol{A}_{3} \cdot \boldsymbol{k}_{2}\right)\left(\boldsymbol{A}_{2}^{*} \cdot \boldsymbol{k}_{2}\right)\right|^{2}\right\} .
\end{aligned}
$$

The common factor $\delta\left(\Omega_{2}-\Omega_{3}\right) n\left(\boldsymbol{k}_{2}\right) n\left(\boldsymbol{k}_{3}\right)$ tells that we have here the contribution of two pairs of waves with the same frequencies. All three terms are generally nonzero (and positive) when the dispersion law is nonmonotonic or non-isotropic so that $\Omega_{2}=\Omega_{3}$ does not require $k_{2}=k_{3}$. In most interesting cases, however, $\Omega_{k}$ is a monotonous function of the modulus $k$ so that $k_{2}=k_{3}$. Let us show first that wave interaction does not contribute $\lambda$ in this case. Indeed, the first two terms, (3/4), that came out of the first two terms of (2), are proportional to the difference, $\boldsymbol{V}_{213}^{*}-\boldsymbol{V}_{3-12}$, between the amplitude of decay into a wave with $\boldsymbol{k}_{1}$ and confluence with a wave with $-\boldsymbol{k}_{1}$. Interaction coefficients for $k_{2}=k_{3}$ have rotational symmetry and are thus functions of wavenumbers so that $V_{213}-V_{3-12}=V_{213}-V_{312}=V_{212}-V_{212}=0$.

The last term (5) comes from the last two terms of (2) and does not contain the interaction coefficient $V$. This term is due to nonlinear relation between Eulerian and Lagrangian variables rather than due to wave interaction. We can compare (5) with the growth rate of the squared density for non-interacting waves, see (12) in [8] written there in terms of the energy spectrum, $E^{\alpha \beta}(\boldsymbol{k}, \omega)=$ $2 \pi A^{\alpha}(\boldsymbol{k}) A^{* \beta}(\boldsymbol{k})\left[n(\boldsymbol{k}) \delta\left(\omega-\Omega_{\boldsymbol{k}}\right)+n(-\boldsymbol{k}) \delta\left(\omega+\Omega_{-\boldsymbol{k}}\right)\right]$. The comparison shows this part of our logarithmic growth rate being exactly half the growth rate for the second moment as it should be for a short-correlated flow [2]. Indeed, the process of creation of density inhomogeneities is effectively short-correlated since the time it takes $\left(1 / \Omega_{k} \epsilon^{4}\right.$ or longer) exceeds the correlation time of velocity divergence in the Lagrangian frame, $1 / \Omega_{k}$. For monotonous $\Omega(k)$, (5D) is nonzero only if the polarization vector $\boldsymbol{A}_{\boldsymbol{k}}$ is neither parallel nor perpendicular to $\boldsymbol{k}$ i.e. contains both solenoidal and potential components. This is not the case for most waves in continuous media. We thus conclude that for most common situations (in particular, for sound or surface waves) the entropy production rate is zero in the order $\epsilon^{4}$. Note that for surface waves, the canonical variables are elevation $\eta(\boldsymbol{r}, t)$ and the potential $\phi(\boldsymbol{r}, z=\eta, t)$ which are related to the surface velocity by a nonlinear relation $\boldsymbol{v}=\nabla \phi(\boldsymbol{r}, \eta, t)$. Expanding it in the powers of $\eta$, one can show that this extra nonlinearity does not contribute $\lambda$ in the order $\epsilon^{4}[25]$. We find it remarkable that the flow of random potential waves is only weakly compressible (i.e. the senior Lyapunov exponent is much larger than the sum of the exponents).

Therefore, we consider now the clustering rate in the presence of solenoidal currents and potential waves, the situation most relevant for oceanological applications [26]. Consider the solenoidal flow $\boldsymbol{u}$ weakly perturbed by potential waves with $\boldsymbol{v}$. To derive $\lambda$ in the lowest (second) order in $\epsilon=k v / \Omega_{k}$, we neglect the contribution of $\boldsymbol{v}$ into $\boldsymbol{X}$ in (11) and assume $\partial_{t} \boldsymbol{X}(t, \boldsymbol{x}) \approx \boldsymbol{u}(t, \boldsymbol{X}(t, \boldsymbol{x}))$. In this order, $w=\nabla \cdot \boldsymbol{v}$ is Gaussian and one may integrate by parts: $\langle w(0, \boldsymbol{x}) w(t, \boldsymbol{X}(t, \boldsymbol{x}))\rangle=\int d t^{\prime} d \boldsymbol{x}^{\prime} \Phi\left(t^{\prime}, \boldsymbol{x}^{\prime}-\right.$ $\boldsymbol{x})\left\langle\delta w(t, \boldsymbol{X}(t, \boldsymbol{x})) / \delta w\left(t^{\prime}, \boldsymbol{x}^{\prime}\right)\right\rangle$. Here $\Phi\left(t^{\prime}-t, \boldsymbol{x}^{\prime}-\boldsymbol{x}\right)=$ $\left\langle w(t, \boldsymbol{x}) w\left(t^{\prime}, \boldsymbol{x}^{\prime}\right)\right\rangle$ is the Eulerian correlation function and

$$
\lambda \approx \int_{0}^{\infty} d t\langle\Phi[t, \boldsymbol{J}(t)]\rangle, \quad \boldsymbol{J}(t) \equiv \boldsymbol{X}(t, \boldsymbol{x})-\boldsymbol{x} .
$$

Waves and currents are considered statistically independent in this order. Using the spectrum, $k^{\alpha} k^{\beta} E_{\boldsymbol{k}}^{\alpha \beta} \equiv$ $k^{2} E_{\boldsymbol{k}}$, we can express $\Phi(t, \boldsymbol{r})=(2 \pi)^{-d} \int k^{2} E_{\boldsymbol{k}} \cos (\boldsymbol{k} \cdot \boldsymbol{r}-$ $\left.\Omega_{\boldsymbol{k}} t\right) d \boldsymbol{k}$ and rewrite (6) as a weighted spectral integral:

$$
\begin{aligned}
& \lambda=(2 \pi)^{-d} \int k^{2} E_{\boldsymbol{k}} \mu(\boldsymbol{k}) d \boldsymbol{k}, \\
& \mu(\boldsymbol{k})=\int_{0}^{\infty}\left\langle\cos \left[\boldsymbol{k} \cdot \boldsymbol{J}(t)-\Omega_{\boldsymbol{k}} t\right]\right\rangle d t .
\end{aligned}
$$

The spectral weight $\mu(k)$ is the Lagrangian correlation time of the $k$-harmonic of $w$ and is expressed via the characteristic function of the particle drift $\boldsymbol{J}(t)$. Without currents, (78) reproduce the first term of (2) since only the zero-frequency wave contributes. Already a steady uniform current $\overline{\boldsymbol{u}}$ contributes the clustering rate in the order $\epsilon^{2}$ if there are waves whose 
Doppler-shifted frequency is zero in the current reference frame: $\lambda=(2 \pi)^{-d} \int k^{2} E_{\boldsymbol{k}} \delta\left(\Omega_{\boldsymbol{k}}-\boldsymbol{k} \cdot \overline{\boldsymbol{u}}\right) d \boldsymbol{k}$. Similar Cherenkov resonance has been noticed before for diffusivity [27]. In the rest of the paper we consider the fluctuating part of current velocity characterized by the rms velocity $u_{0}^{2} \equiv\left\langle u^{2}\right\rangle$ and the correlation time $\tau \equiv \int\left\langle u_{\alpha}(0, \boldsymbol{x}) u_{\alpha}(t, \boldsymbol{X}(t, \boldsymbol{x})\rangle d t / u_{0}^{2}\right.$. Accordingly, there are two dimensionless parameters that describe spatial and temporal relationships between wave and current parameters respectively: $L \equiv k u_{0} \tau$ is the ratio between the distance passed by the fluid particle during $\tau$ and the wavelength, and $T \equiv \Omega_{\boldsymbol{k}} \tau$ is the ratio between the correlation time of currents and wave period. The characteristic function $\langle\exp [i \boldsymbol{k} \cdot \boldsymbol{J}(t)]\rangle$ generally depends on the details of the currents statistics but it has universal behavior both at $t \ll \tau$ and $t \gg \tau$ where general calculations are possible. On the plane of the dimensionless parameters $L, T$ we distinguish three regions of different asymptotic behavior.

Consider first the ballistic limit when the integral (8) is determined by the times $t \ll \tau$ when the drift velocity does not change and $\boldsymbol{J}(t) \approx \boldsymbol{u}(0, \boldsymbol{x}) t$. Again, only those waves contribute that are in a Cherenkov resonance with the current (whose phase velocity coincides with the local projection of the current velocity): $\mu=\pi\left\langle\delta\left(\Omega_{\boldsymbol{k}}-\boldsymbol{k} \cdot \boldsymbol{u}\right)\right\rangle$. In this limit, the weight $\mu$ is determined by the single-time probability distribution of the current velocity which we denote $\mathcal{P}(\boldsymbol{u})$. In particular, for the isotropic Gaussian $\mathcal{P}(u) \propto \exp \left(-u^{2} / 2 u_{0}^{2}\right)$, we get

$$
\mu(k)=(\pi d / 2)^{1 / 2}\left(k u_{0}\right)^{-1} \exp \left[-\left(\sqrt{d} \Omega_{k} / \sqrt{2} k u_{0}\right)^{2}\right],
$$

The ballistic approximation and (9) hold when $k^{2} u_{0}^{2} \tau^{2} / d$ is much larger than both unity and $\Omega_{k} \tau$.

The second universal limit is that of a slow clustering which proceeds for the time exceeding the correlation time of currents. At $t \gg \tau$, we use the diffusion approximation, $\langle\exp [i \boldsymbol{k} \cdot \boldsymbol{J}(t)]\rangle=\exp \left[-k^{2} u_{0}^{2} \tau t / d\right]$, in (8):

$$
\mu(k)=\tau \frac{d\left(k u_{0} \tau\right)^{2}}{\left(k u_{0} \tau\right)^{4}+\left(d \Omega_{k} \tau\right)^{2}} .
$$

That answer and the diffusive approximation hold when both $k^{2} u_{0}^{2} \tau^{2} / d$ and $\Omega_{k} \tau$ are small. Formulas (7/10) can be compared with the expression for the clustering rate for waves with a linear damping, $\lambda \simeq \int k^{2} E_{k} \gamma_{k}\left(\Omega_{k}^{2}+\right.$ $\left.\gamma_{k}^{2}\right)^{-1} d \boldsymbol{k}$ [24]. We see that in this limit the diffusive motion of fluid particles due to currents is equivalent in its effect to a damping of waves with $\gamma_{k}=k^{2} u_{0}^{2} \tau / d$, where $u_{0}^{2} \tau / d$ is the eddy diffusivity.

The third asymptotic regime takes place for fastoscillating waves when $\Omega_{k} \tau$ exceeds both unity and $k^{2} u_{0}^{2} \tau^{2} / d$. An integral of the fast oscillating exponent with a slow function, $\int_{0}^{\infty} \cos \left(\Omega_{k} t\right) f(t) d t$, decays as $\Omega_{k}^{-2 n-2}$ where $2 n+1$ is the lowest order of the nonvanishing derivative of $f(t)=\langle\exp [i \boldsymbol{k} \cdot \boldsymbol{J}(t)]\rangle$ at $t=0$. When all odd derivatives at zero are zero, the integral decays exponentially. We see that the answer depends on the details of the statistics of currents.

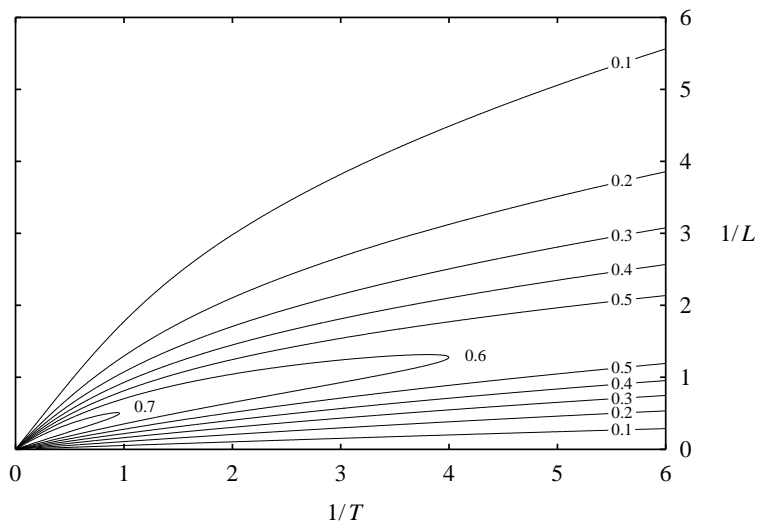

FIG. 1: The isolines of the dimensionless clustering rate $\mu(k) \Omega_{k}$ given by (11), here $L=k u_{0} \tau, T=\Omega_{k} \tau$.

If $\boldsymbol{u}(t, \boldsymbol{X}(t, \boldsymbol{x}))$ is Gaussian and isotropic with $\left\langle u^{\alpha}(0, \boldsymbol{x}) u^{\beta}(t, \boldsymbol{X}(t, \boldsymbol{x}))\right\rangle=\left(u_{0}^{2} / d\right) \delta^{\alpha \beta} \exp (-|t| / \tau)$ then

$$
\mu(k)=\tau \int_{0}^{\infty} d s \cos (T s) \exp \left[\left(L^{2} / d\right)\left(1-s-e^{-s}\right)\right] .
$$

It gives both limits (910) and

$$
\mu(k)=\left(k u_{0}\right)^{2} / \tau \Omega_{k}^{4} d,
$$

at large $\Omega_{k}$ since the lowest non-vanishing derivative is $f^{\prime \prime \prime}(0)$. Isolines of (11) are shown in Figure 1 for arbitrary parameters. Remind that the whole description based on (6) is valid when $v \ll u$.

If one interpolates between the ballistic and diffusive regimes (i.e. between $J^{2} \propto t^{2}$ and $J^{2} \propto t$ ) with the help of the function $\sqrt{1+(t / \tau)^{2}}-1$, which is smooth at $t=0$, then the weight factor can be calculated analytically:

$$
\begin{aligned}
& \mu(k)=\tau \int_{0}^{\infty} d s \cos (T s) \exp \left[\left(L^{2} / d\right)\left(\sqrt{1+s^{2}}-1\right)\right] \\
& =\frac{\tau L^{2}}{d} \exp \left(\frac{L^{2}}{d}\right) \frac{K_{1}\left(\sqrt{\left(L^{4} / d^{2}\right)+T^{2}}\right)}{\sqrt{L^{4} / d^{2}+T^{2}}}
\end{aligned}
$$

Here $K_{1}(x)$ is a Bessel function of an imaginary argument having the following asymptotics: $K_{1}(x)=$ $\sqrt{\pi / 2 x} \exp (-x)[1+\mathcal{O}(1 / x)]$ for $x \gg 1$ and $K_{1}(x) \simeq$ $1 / x+\mathcal{O}(x \ln (x))$ for $x \ll 1$. We see that (13) reproduces (9, 10) in the regions $L^{2} / d \gg 1, L^{2} / d \gg T$ and $L^{2} / d \ll 1, T \ll 1$ respectively. At the fast-oscillation limit one gets an exponentially small contribution

$$
\mu(k)=\tau \sqrt{\frac{\pi\left(k u_{0}\right)^{4} \tau}{2 d^{2} \Omega_{k}^{3}}} e^{-\Omega_{k} \tau} .
$$

That concludes the analysis of the weight $\mu(k)$ and we can now turn to (77) to get the clustering rate $\lambda$.

When the wave spectrum is not very wide (with the width comparable to $k$ ) we get

$$
\lambda \simeq(k v)^{2} \mu(k)=\epsilon^{2} \Omega_{k}^{2} \mu(k) .
$$


Let us now find out which wavenumbers contribute (7) when the spectrum is wide. Consider an isotropic power spectrum $E_{k} \propto k^{b-d}$ between some $k_{\min }$ and $k_{\max }$ and the dispersion relations $\Omega_{k}=C k^{a}$ 23]. Consider first the ballistic regime. For $(a>1 \wedge b>0) \vee(a<1 \wedge b<0)$ the wavenumber $k_{*}=\left[b u_{0}^{2} / d C^{2}(a-1)\right]^{1 /(2 a-2)}$ determines $\lambda$. For $(b \leq 0 \wedge a>1) \vee(b<-1 \wedge a=1)$ the clustering rate is determined by $k_{\text {min }}$, while for $(b \geq 0 \wedge a<1) \vee(b \geq-1 \wedge a=1)$ by $k_{\max }$. Let us give physical examples using Kolmogorov spectra of waves. For capillary waves on a deep water, $\Omega_{k} \propto k^{3 / 2}$ and $E_{k} \propto k^{-11 / 4}$, and $\lambda$ is determined by $k_{\text {min }}$ i.e. by longest waves in the wave turbulent spectrum (assuming the ballistic approximation is valid for them). For gravity waves on a deep water, $\Omega_{k} \propto k^{1 / 2}$, and for both Kolmogorov solutions, $E_{k} \propto k^{-20 / 6}$ and $E_{k} \propto k^{-7 / 2}$, the clustering rate is determined by waves around $k_{*}$. For diffusive regime, the clustering rate is determined by $k_{\max }$ if $b \geq \max [2 a-4,0]$ and by $k_{\min }$ if $b<\max [2 a-4,0]$.

Estimates (910]12]14) show that $\lambda /\left(\epsilon^{2} \Omega_{k}\right) \simeq \Omega_{k} \mu(k)$ is a dimensionless function which has a maximum of order unity either in the ballistic regime where the phase velocity of wave is comparable to the current velocity or in the diffusive regime where the eddy diffusivity $u_{0}^{2} \tau$ is comparable to $\Omega_{\boldsymbol{k}} k^{-2}$ (in the third asymptotic regime $\lambda /\left(\epsilon^{2} \Omega_{k}\right)$ is always small). In those cases, $\lambda / \Omega_{k} \simeq \epsilon^{2}$, i.e. the degree of clustering during a period is the squared wave nonlinearity (typically $\epsilon$ is between 0.1 and 0.01 ). Such clustering is pretty fast (minutes for meter-sized gravity waves and a week for fifty-kilometer-sized inertio-gravity waves). Therefore, it is likely that the interplay between waves and currents is the source of inhomogeneities of floater distribution in many environmental situations.

Clustering leads to fractal distribution of floaters over the surface. When compressible component of the velocity is small, the Lyapunov exponents are due to the current flow, $\lambda_{1} \sim \lambda_{2} \sim \tau^{-1}$. Then, the fractal dimension of the density distribution can be expressed by the KaplanYorke formula $1+\lambda_{1} /\left|\lambda_{2}\right|=2-\lambda /\left|\lambda_{2}\right| \approx 2-\lambda \tau$. The fractal part reaches maximum in the ballistic regime when $\Omega_{k} \simeq k u_{0}$, then $\lambda \tau \simeq \epsilon^{2} \Omega_{k} \tau=\epsilon^{2} k \ell$ grows with $\ell=u_{0} \tau$ and reaches order unity when $k \ell \simeq \epsilon^{-2}$. Therefore, the distribution is most fractal when waves are weak and short while currents are long and strong. For example, meter-sized gravity waves on water surface will produce most inhomogeneous distribution of floaters when there are currents with velocities in meters per second and scales in hundreds of meters. We see that the currentto-wave ratio of scales, $k \ell$, compensates for a small wave nonlinearity, $\epsilon^{2}$, so that even weak waves with the help of solenoidal currents can produce very inhomogeneous fractal distribution of matter.

As a final remark, note that apart from fluid mechanics, one can think about the evolution of a dynamical system as a flow in the phase space and treat density as a measure. Solenoidal (incompressible) flow corresponds to Hamiltonian dynamics and to a constant (equilibrium) measure. Compressibility corresponds to pumping and damping i.e. to non-equilibrium. Indeed, the notion of singular (fractal) measures first appeared in nonequilibrium statistical physics [17, 18, 19] and then was applied in fluid mechanics 2, 3, 6, 11, 12]. Therefore, the formulas (6-14) also describe the entropy production rate in dynamical systems under the action of perturbations periodic in space and in time.

The work was supported by the ISF. We thank V. Lebedev and E. Tziperman for helpful explanations.
[1] K. Herterich and K. Hasselmann, J. Phys. Oceanogr. 12, 704 (1982).

[2] G. Falkovich, K. Gawȩdzki, and M. Vergassola, Rev. Mod. Phys. 73, 913 (2001).

[3] E. Balkovsky, G. Falkovich, and A. Fouxon, Phys. Rev. Lett. 86, 2790 (2001).

[4] D. Ruelle, J. Stat. Phys. 95, 21 (1999).

[5] G. Falkovich and A. Fouxon, New J. Physics 6 (2004).

[6] J. Bec, K. Gawȩdzki, and P. Horvai, Phys. Rev. Lett. 92, 224501 (2004).

[7] A. Balk and R. McLaughlin, Phys. Lett. A 256, 299 (1999).

[8] A. M. Balk, G. Falkovich, and M. G. Stepanov, Phys. Rev. Lett. 92, 244504 (2004).

[9] B. Eckhardt and J. Schumacher, Phys. Rev. E 64, 016314 (2001).

[10] D. B. R. Ramshankar and J. Gollub, Phys. Fluids A 2, 1955 (1990).

[11] J. C. Sommerer and E. Ott, Science 259, 335 (1993).

[12] J. C. Sommerer, Phys. Fluids 8, 2441 (1996).

[13] A. Nameson, T. Antonsen, and E. Ott, Phys. Fluids 8, 2426 (1996).

[14] E. Schroder et al, Phys. Rev. Lett. 76, 4717 (1996).
[15] J. R. Cressman and W. Goldburg, J. Stat. Phys. 113, 875 (2003).

[16] P. Denissenko, G. Falkovich, and S. Lukaschuk, Phys. Rev. Lett. 97 (2006), nlin.CD/0511044.

[17] R. Bowen and D. Ruelle, Invent. Math. 29, 181 (1975).

[18] Y. G. Sinai, Russian Math. Surveys 27, 21 (1999).

[19] J. Dorfman, Introduction to Chaos in Nonequilibrium Statistical Mechanics (Cambridge Univ. Press, 1999).

[20] J. R. Cressman, J. Davoudi, W. Goldburg, and J. Schumacher, New J. Physics 6, 53 (2004).

[21] G. Falkovich and A. Fouxon, nlin.CD/0312033.

[22] T. Yamada and K. Kawasaki, Progr. Theor. Phys. 38, 1031 (1967).

[23] V. E. Zakharov, V. L'vov, and G. Falkovich, Kolmogorov spectra of turbulence (Springer-Verlag, Berlin, 1992).

[24] G. Falkovich and D. Shlomo, Phys. Rev. E 71, 067304 (2005).

[25] I. Fouxon and M. Vucelja, in preparation.

[26] J. Pedlosky, Geophysical fluid dynamics (Springer, New York, 1987).

[27] A. M. Balk, Private communication (2005). 